\title{
Prácticas comunitarias del desarrollo: una mirada desde los oficios y el trabajo*
}

\author{
Andrés Roldán Tonioni**
}

Resumen: Se describen las prácticas artesanales de una comunidad de oficio de la Novena Región de la Araucanía, Chile, a través de las cuales se construye una noción del desarrollo. Metodológicamente se utilizó la teoría fundamentada mediante el modelo propuesto por Glaser y Strauss (1967) y el uso del software Atlas ti para apoyar el análisis de los datos. Los resultados revelan que la práctica artesanal es un trabajo que se posiciona en la tensión entre el rescate cultural y ancestral de una práctica tradicional y los requerimientos de una economía de acumulación y de conversión del trabajo en capital. La construcción de significados de los artesanos de la región se caracteriza por dar cuenta de subjetividades alternativas a los modelos discursivos dominantes del desarrollo. Se presenta una propuesta de análisis de los oficios, a través de tres ejes de análisis: el trabajo, el oficio y el saber. Trabajo.

Palabras clave: Prácticas artesanales, Comunidades de oficio, Desarrollo,

\section{Communitarian practices on development: A perspective from handcraft skills and work}

\begin{abstract}
The handcrafted practices of a occupation community of the Ninth Region of the Araucania (Chile) are described, through which a notion of the development is built. Methodologically speaking the Grounded Theory by means of the pattern proposed by Glaser \& Strauss (1967) and the software Atlas ti support the analysis of the data was utilized. The results reveal that the handmade practice is a job that is positioned in the tension between the cultural and ancestral rescue of a traditional practice and the requirements of an economy of accumulation and conversion of work in capital. The construction of meanings of the craftsmen in the region is characterized by taking into account alternate subjectivities to the dominant discursive models of development. A proposal of analysis of the occupations is presented, through three analysis axes: the job, the occupation and the knowledge.
\end{abstract}

Key words: Handcraft practices, handcraft skills Communities, Development, Work.

\footnotetext{
* La presente publicación da a conocer la investigación social denominada: Prácticas comunitarias del desarrollo: una mirada desde los oficios y el trabajo, realizada en el marco de obtención del grado académico de Magíster en Psicología, mención Psicología Comunitaria de la Universidad de Chile, llevada a cabo el año 2008.

** Universidad Católica de Temuco, Temuco, Chile. Email: aroldan@uct.cl
} 


\section{Práticas comunitárias do desenvolvimento: Uma mirada desde os oficios e o trabalho}

Resumo: Descrevem-se as práticas artesanais de uma comunidade de oficio da Nona Região da Araucanía, Chile, mediante as quais se constrói uma noção do desenvolvimento. Metodologicamente utilizou-se a teoria fundamentada mediante o modelo proposto por Glaser e Strauss (1967) e o uso do software Atlas ti para apoiar o exame dos dados. Os resultados revelam que a prática artesanal é um trabalho que se posiciona na tensão entre o resgate cultural e ancestral de uma prática tradicional e os requerimientos de uma economia de acumulação e de conversão do trabalho em capital. A construção de significados dos artesãos da região caracteriza-se por dar conta de subjetividades alternativas aos modelos discursivos dominantes do desenvolvimento. Apresenta-se uma proposta de análise dos oficios, através de três eixos de análises: o trabalho, o oficio e o saber.

Palavras-chave: práticas artesanais, comunidades de oficio, desenvolvimento, trabalho.

Recibido: 01.06.2009

Aceptado: 05.08.2009

$* * *$

\section{El problema y su encuadre teórico}

Asumimos el desarrollo como discurso producido históricamente ${ }^{1}$, lo que implica indagar en las razones que tuvieron tantos países para comenzar a considerarse subdesarrollados a comienzos de la segunda posguerra. De esta forma, Escobar (1998: 31) habla del desarrollo como "una experiencia históricamente singular, como la creación de un dominio del pensamiento, y de la acción”2. La configuración del escenario internacio-

\footnotetext{
${ }^{1}$ Para Sunkel y Paz (citado en Boisier, 2001) “El desarrollo es un tópico de la posguerra y habría que agregar es un tópico de las Naciones Unidas. Ya en la carta del Atlántico firmada en 1941 por Churchill y Roosvelt se expresa que el único fundamento cierto de la paz reside en que todos los hombres libres del mundo puedan disfrutar de seguridad económica y social y por lo tanto, se comprometen a buscar un orden mundial que permita alcanzar estos objetivos una vez finalizada la guerra”. Por lo tanto, no es de extrañar que la CEPAL, comisión de las naciones unidas, hiciera del análisis del desarrollo un tema preferente tanto en la reflexión como los estudios empíricos hasta el día de hoy. Desde mi punto de vista, ese es el modelo de desarrollo dominante que se ha tratado de imponer y que hoy ha sido encauzado por el proyecto político, ideológico y económico neoliberal.

${ }^{2}$ En otro de sus textos Escobar (2005: 18), señala que las principales orientaciones teóricas asociadas a la Escuela Latinoamericana del Desarrollo pueden ser descritas de la siguiente manera: "A lo largo de los últimos cincuenta años, la conceptualización sobre el desarrollo en las ciencias sociales ha visto tres momentos principales correspondientes a tres orientaciones teóricas contrastantes: la teoría de la modernización en las décadas de los cincuenta y sesenta, con sus teorías aliadas de crecimiento y desarrollo; la teoría de la dependencia y perspectivas relacionadas en los años sesenta y setenta; y aproximaciones críticas al desarrollo como discurso cultural en la segunda mitad de la década de los ochenta y los años noventa”, y continúa luego, "Los tres momentos mencionados pueden ser clasificados de acuerdo con los paradigmas originarios de los cuales emergieron: teorías liberales, marxistas y postestructuralistas, respectivamente"
} 
nal, en este contexto discursivo e histórico, se ha cristalizado también a escala territorial, en materia de atraso económico y dominación cultural.

Según lo anterior, creemos necesario hoy día relevar diferentes discursos y representaciones que no se encuentren tan mediados por la construcción del desarrollo, o sea, la necesidad de cambiar las prácticas de saber y hacer, la necesidad de multiplicar centros y agentes de producción de conocimientos, particularmente, hacer visibles las formas de conocimiento producidas por aquéllos quienes supuestamente son los “objetos" del desarrollo para que puedan transformarse en sujetos y agentes; dos maneras especialmente útiles de lograrlo son: primero, enfocarse en las adaptaciones, subversiones y resistencias que, localmente, la gente efectúa en relación con las intervenciones del desarrollo; y, segundo, destacar las estrategias alternas producidas por movimientos sociales al encontrarse con proyectos de desarrollo (Escobar, 2005).

Ahora, estas subversiones operan en la estructura a través de un cúmulo de acciones de intervención para la gestión de mejoras de vida comunitarias que denominaremos como: prácticas comunitarias (Isla \& Colmegna, 2007). Las prácticas sociales o comunitarias son las actividades que operan transformaciones a partir de saberes en movimiento, las prácticas son la experiencia, lo vivido, lo realmente sucedido (Martínez, 2006), constituyen la compleja articulación de relaciones sociales, políticas y económicas entre actores que se movilizan en un campo de relaciones de poder.

Transitamos en la búsqueda de estas nuevas representaciones respecto el desarrollo, pero tal como plantea Elizalde (2005) el desarrollo es el despliegue de un proceso multidimensional, y querer abarcarlo en su totalidad resultaría una tarea demasiado ambiciosa. En esta investigación se abordó el desarrollo desde la dimensión del trabajo debido a las características propias de nuestros sujetos de investigación, sosteniendo que hay distintas formas de concebir a éste (el trabajo) desde las comunidades y que esas formas se reflejan en subjetividades y prácticas específicas y alternativas a las lógicas de producción dominantes. En este sentido, partimos de la certeza de que en la cotidianeidad emergen diversas maneras de hacer, que eluden a las formas de dominación colonial principalmente en los planos político, económico y cultural, como micro resistencias movilizadas por prácticas creativas de transformación de lo dado. A estas prácticas de resistencia Certeau (1996) las denomina como artes del hacer, aquellas que organizan nuestra vida cotidiana.

Este posicionamiento teórico, nos instan a buscar nuevas miradas, a la sensibilización de categorías, a comprender el proceso de trabajo más allá de la producción y el consumo, entendiendo que si bien por todos lados se extiende y se reproduce la lógica colonial de reproducción del orden económico y cultural (Castro Gómez \& Grosfoguel, 2007: Bhabha, 1994), resulta tanto más urgente destacar cómo una sociedad entera, o parte de ésta, no se reduce a ella. Para los fines de esta presentación, y por ser una buena aproximación inicial, entenderemos el trabajo como aquella actividad humana que comienza a pen- 
sarse con su negación. Esto significa que la piedra de toque a partir de la cual el trabajo penetra en distintos ámbitos de la especulación, es la idea de la alienación del trabajo o trabajo alienado (Hopenhayn, 2001).

La preocupación, entonces, es la redefinición del modelo de desarrollo $^{3}$ (Garretón, 2000). Según esto, la investigación desde sus comienzos se aparta de la concepción del desarrollo como transformaciones hacia una economía de mercado, como hasta el día de hoy se ha pensado esta categoría, o sea, el modelo de desarrollo orientado a la estabilización económica, la apertura al exterior, la desregulación de los mercados y la privatización (y transnacionalización) de los procesos productivos (Di Filippo, 2007). El desarrollo en América Latina, desde este enfoque, debe ser entendido y comprendido en base al proceso de cómo las economías se vincularon históricamente, y en la actualidad, al mercado mundial y la forma en que sobreviven discursos coloniales que perpetúan la dominación de unas regiones o grupos sobre otros. Y nuestro interés parte de encontrar nuevos saberes y prácticas que permitan modelos pertinentes a la región, desde una plataforma de desarrollo, describiendo y relevando la capacidad creativa y de acción de los sujetos reconocidos como artesanos.

Según las políticas de planificación territorial, la Araucanía se divide en cuatro grandes territorios económicos: Araucanía Andina, Cluster Forestal, Valle Araucanía, Vergel del Sur. Esta investigación se inserta en el territorio Valle Araucanía que comprende las comunas de: Temuco, Nueva Imperial, Chol Chol, Freire y Padre Las Casas. Y que está constituida por tres mesas intercomunales de pequeños productores, divididas por sector: La Mesa de Mueble y Madera, La Mesa de Artesanía y La Mesa de la Agricultura.

\section{Mapa de la IX Región de la Araucanía, Valle Araucanía}

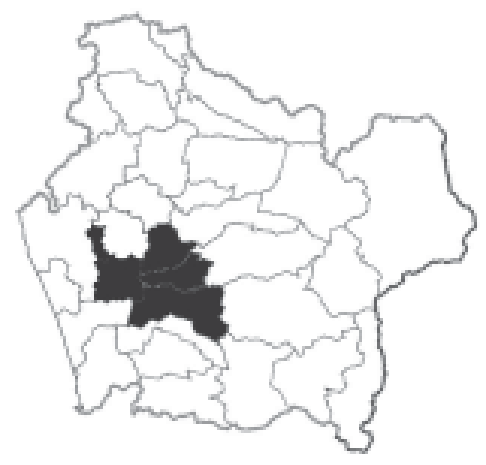

\footnotetext{
${ }^{3}$ En este punto comparto la postura de Manuel Antonio Garretón que plantea que actualmente estamos en un contexto de necesidad de redefinición del modelo de desarrollo en donde debemos ser capaces de comprender dos fenómenos paralelos: La internacionalización de los mercados, proceso que en Chile y América Latina se ha llevado a cabo por la industrialización sustitutiva de importaciones, y por otro lado, el debilitamiento del Estado, que pierde su rol hegemónico como agente del desarrollo, ante la irrupción de enormes empresas supranacionales que instrumentalizan la acción social y política.
} 
Por cercanía profesional, accesibilidad y las características particulares que presentaba se trabajó con La Mesa de Artesanía del territorio Valle Araucanía. Se puede decir que la mayoría de quienes conforman la Mesa de Artesanía son mujeres, en gran número provenientes de sectores rurales y muchas veces también pertenecientes a comunidades Mapuche. A través de la investigación veremos que estos productores conforman una comunidad de oficio, de prácticas socio-comunitarias bien definidas.

Las comunidades de artesanas y artesanos participantes de la Mesa de Artesanías del Valle Araucanía, en la mayoría de los casos, congregan de cinco a diez artesanas que se unen en un trabajo colectivo. Por ejemplo, la agrupación Allilen Zomo Zuwen (mujer que sonríe trabajando), del sector Folilco (Agua de Raíz) de la comuna de Freire, tiene representación en la Mesa de Artesanía a través de una de sus integrantes que viaja a Temuco a las reuniones, pero en sí misma está compuesta por alrededor de diez a doce mujeres artesanas.

La Mesa de Artesanos, de las tres mesas que conforman el territorio Valle Araucanía, fue la última en instituirse como tal. Nace como una iniciativa público-privada a mediados del año 2007 y viene trabajando desde entonces con organismos públicos como el SERCOTEC IX Región, Chile Emprende, FOSIS, entre otros.

Intuíamos, basados en observaciones anteriores de experiencias con estos pequeños productores, que este grupo de artesanos nos brindaría esas representaciones alternativas del desarrollo, desde la perspectiva del traba$\mathrm{jo}^{4}$. Se considera a los artesanos como agentes protagonistas de la transformación social a nivel local y de esta forma ahondaremos, a través de sus prácticas de trabajo, en la construcción significativa del desarrollo.

Estos actores sociales que identificamos como pequeños productores, constituyen uno de motores principales del empleo regional, su actuar principal se basa en los lazos que se establecen entre unos y otros para la consecución de fines, por tanto, son agentes que se movilizan por transformaciones sociales en campos de poder bien definidos. El problema del desarrollo comunitario es el de superación del estado de estancamiento, de no cambio o no transformación, de la comunidad. Es en las pequeñas empresas en donde más tardan en operar las transformaciones tendientes a optimizar la comunidad de práctica que constituyen (Martínez, 2006).

Según lo anterior, la pregunta de investigación fue: ¿Cuál es el significado atribuido por los artesanos de la Región de la Araucanía a sus prácticas sociales del trabajo y los oficios a través del cual construyen una

\footnotetext{
${ }^{4}$ Según Hopenhayn "la modalidad de trabajo cambia sustancialmente con el advenimiento de la revolución industrial, y la visión que el artesano tenía de su trabajo debió verse distorsionada por la nueva división del trabajo, el régimen de asalariados y la inseguridad en el empleo, rasgos que contrastaban en forma aguda con el estilo de trabajo de los gremios corporativos" (2001: 17)
} 
noción acerca del desarrollo? A la que se respondió a través de los siguientes objetivos específicos: 1. Identificar las principales prácticas sociales del trabajo y los oficios que cotidianamente reproducen el quehacer de los artesanos de la mesa de artesanía del Valle Araucanía; 2. Determinar el significado otorgado a las prácticas sociales de trabajo y los oficios de los artesanos a través de los discursos en torno a ellas; 3. Aproximarnos a la noción de desarrollo que construyen estos pequeños productores regionales, en tanto supuestos que regulan sus prácticas de transformación sociocomunitarias, y 4. Contribuir a la reflexión sobre el desarrollo a partir de la experiencia generada por las prácticas sociales de la comunidad de artesanos.

\section{El contexto local}

La Región de la Araucanía es una de las zonas donde los indicadores de pobreza superan el promedio nacional (13,7\%), ya que el índice de pobreza presentado por la región es de un 20,1\% sólo superado por la VIII Región que presenta un 20,7\% (MIDEPLAN, 2006). Se está hablando de una de las regiones con mayores índices de pobreza a nivel nacional, lo que impacta directamente en la estructura social. Lo anterior se refuerza debido a que, según las cifras, la región es la penúltima en el ranking nacional de Desarrollo Humano en Chile (MIDEPLAN, PNUD, 2000).

Su composición étnica la presenta como una de las regiones en donde se concentra la mayor parte de la población reconocida como descendiente de pueblos originarios, en este caso Mapuche, quienes a nivel nacional suman 604.349 habitantes, de los cuales 203.221 se concentran en la IX Región (23,4\% de la población total regional), seguida por la Región Metropolitana y la X Región (Censo, 2002). Su importancia no sólo radica en su magnitud numérica, sino también en la riqueza sociocultural que le es propia. Sin embargo, pese a este panorama rico en diversidad cultural, la realidad muestra que el pueblo Mapuche sufre directamente de los rumbos que ha asumido el desarrollo en los distintos proyectos políticos provenientes del Estado chileno. Actualmente existen variables que complejizan la configuración étnica de la región, vinculada a la calidad de vida asociada a estos grupos, la ruralidad de sus contextos geográficos, el grado de integración-exclusión a la sociedad chilena, la desigualdad en la distribución y acceso a ingresos, entre otras, ubicándola en una posición de vulnerabilidad social y cultural.

Así, “a nivel nacional se constata que las personas que declaran pertenecer al pueblo mapuche tienen en general menores niveles de logro en desarrollo humano ${ }^{5}$ que los que declaran no pertenecer a ningún pueblo

\footnotetext{
5 “El Índice de Desarrollo Humano (IDH) es un instrumento que intenta ser una aproximación general a los elementos más básicos que forman parte del enfoque (...) se concentra en tres dimensiones básicas universales e interculturalmente valoradas (...): salud, educación e ingresos” (PNUD, UFRO y MIDEPLAN, 2003: 9).
} 
originario (...) la mayor disparidad se aprecia en el ámbito del acceso a recursos materiales, donde tanto la situación de pobreza como los ingresos per cápita son ampliamente menos favorables para aquellos que declaran pertenecer al pueblo mapuche (...) es más, comparando entre regiones, las mayores distancias se expresan en la dimensión ingresos, donde los mapuches de la Región de la Araucanía tienen sólo la mitad de los ingresos per cápita que los de la Metropolitana y duplican su porcentaje de incidencia de la pobreza” (PNUD, UFRO y MIDEPLAN, 2003: 14 - 17).

Las principales actividades económicas que conforman el producto interno bruto de la región son: la agropecuaria-silvícola, donde se destacan los cultivos tradicionales, la ganadería y la producción forestal; la hotelería asociada al turismo, todas con una participación cercana al 20\%, y la industria manufacturera con plantas procesadoras de celulosa, como también industrias de muebles con una participación del $15 \%$. La fuerza de trabajo total de la IX Región es del orden de 363.950 personas. De este total, el 28\% está ocupado en el sector agrícola, seguido por la industria manufacturera y comercio, hoteles y restaurantes, mientras que un 10,2\% es la tasa de desocupación ${ }^{6}$ (MIDEPLAN, 2006). En la misma línea, en la Araucanía un 75,2\% del empleo de la región depende de la micro y pequeña empresa. Las actividades productivas dependen, como en la mayoría de las regiones de nuestro país, fundamentalmente de la utilización de sus recursos naturales y del sector de servicios. Recordemos que, la micro, pequeña y mediana empresa representan más del $90 \%$ del universo de empresas en Chile, aportando (en ventas) en un 16\% de la economía y más de un $70 \%$ del empleo (CiPyME, 2007). Cifra, esta última, crucial en el funcionamiento económico-social del país.

Si consideramos que la tasa de cesantía en la IX Región es de $7,1 \%$ y el de pobreza es de 20,1\% (CASEN, 2006), podemos llegar a inferir que gran parte de la población que cuenta con un empleo en una micro y pequeña empresa no puede superar la condición de pobreza, ya que una considerable parte de la población económicamente activa trabaja en éstas. Las cifras son claras en este sentido, se castiga a las personas que se desempeñan en las unidades económicas de menor tamaño.

\footnotetext{
${ }^{6}$ Se han querido mantener las cifras presentadas en el escrito original de la investigación, obtenidas en las referencias señaladas. Sin embargo en la actualidad, según el Informe Económico Regional emitido por el Instituto Nacional de Estadísticas (2009), las cifras no varían significativamente. La Tasa de Desocupación, el último trimestre Octubre-Diciembre de 2008, fue al igual que entonces, de un 10,2\%. La Fuerza de Trabajo, según este Informe, es de 395.640 personas, de la cual los ocupados alcanzan los 355.240. La tasa de cesantía y de personas que buscan trabajo por primera vez, que en conjunto explican la desocupación, fue de $8,0 \%$ y 2,2\% respectivamente. Respecto a la tasa de desocupación nacional que alcanzó 7,5\%, la tasa de desocupación de La Araucanía fue 2,7 puntos porcentuales superior.
} 


\section{Metodología}

Para ir al abordaje de las significaciones de los artesanos se utilizó una metodología cualitativa fundada en los datos (Valles, 1999), que nos permitió la descripción de las cualidades de la práctica artesanal, relevando las perspectivas comunitarias del desarrollo de los artesanos de la Araucanía. Asumimos que, si bien existen diversos métodos para concretar una investigación de las prácticas comunitarias de desarrollo, "tenemos que aceptar que por lo menos la tarea de conceptualizar alternativas debe incluir un contacto significativo con aquellos cuyas alternativas deben ser investigadas” (Escobar, 1998: 419). Por tanto, privilegiamos la observación participante y directa en los escenarios, los talleres y sedes en el campo y en la ciudad, sus casas y las reuniones de la Mesa del Valle Araucanía.

La opción escogida para la selección de informantes fue enfocarse en un proceso gradual, por fases e intencionado de contacto y llegada a las artesanas y artesanos que nos ilustraron respecto a las prácticas cotidianas de su trabajo, con el fin de profundizar en sus relaciones sociales y analizarlas en discusión con el marco conceptual, lo que se ha denominado como muestreo teórico (Strauss \& Corbin, 1990).

Los instrumentos de recolección de datos principales fueron: la aplicación de doce entrevistas en profundidad semi-estructuradas a las artesanasartesanos, dos entrevistas en profundidad a sujetos expertos ${ }^{7}$, dos grupos focales, la observación en terreno en las comunas y las notas de campo ampliadas de estas salidas y de la participación en las reuniones de la Mesa de Artesanía del Valle Araucanía. Estas técnicas se articularon de tal manera que respondieran más bien a una estrategia coherente y razonada de recolección de datos que nos permitiera una triangulación en la obtención de datos. El principio que se utilizó fue la saturación teórica, contrastamos permanentemente los datos obtenidos a partir de las entrevistas con lo que efectivamente recogimos con los grupos focales. Se entrevistó a los primeros artesanos, luego se realizó el primer grupo focal, seguimos entrevistando y realizamos el segundo grupo focal para finalizar las últimas entrevistas en la comuna de Freire.

En coherencia con lo anterior, para el análisis de datos se utilizó el modelo de la teoría fundamentada, con apoyo del software de análisis de datos cualitativo Atlas ti, basada en el método comparativo constante (Glaser \& Strauss, 1967), y los pasos seguidos en esta investigación fueron: De los datos brutos a la codificación inicial o categorización abierta ("open coding”): Su objetivo fue abrir el trabajo con los datos a partir de un proceso de categorización inicial. El desarrollo de las categorías iniciales o codi-

\footnotetext{
${ }^{7}$ Éstos fueron: el Sacerdote y Teólogo Ronaldo Muñoz y el sociólogo Antonio Elizalde. Ambos fueron consultados en su calidad de expertos en torno a la temática del desarrollo, desde la perspectiva de la teología de la liberación y desde la perspectiva del Desarrollo a Escala Humana y sus proyecciones en la actualidad, respectivamente.
} 
ficación axial (“axial coding”): En este momento buscamos propiedades, en pos de estructurar la información, y lo que hicimos fue relacionar las categorías iniciales. Como última operación, la codificación selectiva ("selective coding”), escogimos una categoría como central y luego relacionamos las otras a esa categoría, organizando en forma creciente la articulación de los componentes de la teoría, en un proceso de densificación creciente (Mella, 2003).

\section{Resultados}

"El mayor tiempo que uno trabaja es en la casa porque allá es donde tenemos nuestras cosas, aunque dedicamos algunos tiempos no más, ya que también debemos preocuparnos de las labores de la casa, trabajar en las huertas, con las aves, animales, entonces dedicamos ratos, pero en esos ratos somos felices” (Artesanas de Temuco, grupo focal)

"Me agrada porque es un trabajo que perteneció históricamente a nuestro pueblo, yo genero productos de significación cultural, patrimonial”. (Artesano de Freire)

Uno de los hallazgos principales que arrojó la presente investigación es que la artesanía es un trabajo que se resiste a la alienación, la homogeneización y estandarización de los procesos productivos y del trabajo moderno. Afirmamos que la construcción de significados de los artesanos de la región se caracteriza por dar cuenta de subjetividades alternativas a los modelos discursivos dominantes del desarrollo.

La construcción de significados respecto al desarrollo, desde el ámbito de su práctica artesanal, se despliega desde el rescate cultural. Práctica social que conecta con una cultura ancestral, la Mapuche, pilar clave de la arquitectura comunitaria en la que construyen su pertenencia. Los resultados revelan que la práctica artesanal es un trabajo que se posiciona en la tensión entre el rescate cultural y ancestral de una práctica tradicional y los requerimientos de una economía de acumulación y de conversión del trabajo en capital, que les exige posicionar sus piezas en el mercado regional.

Se presenta una propuesta de análisis de los oficios, a través de tres ejes de análisis: el trabajo, el oficio y el saber. El trabajo como la actividad de participar en un proceso de producción; el oficio, como la 'profesión' aprehendida y desempeñada, la formación en alguna materia; y el saber como el 'arte' del manejo de técnica y el instrumento.

Para los sujetos de investigación, el trabajo artesanal es aquel ejercicio a través del cual se 'ganan' la vida, que realizan con productos naturales, el que les brinda la libertad tanto para ajustar y autorregular sus horarios como para innovar y probar distintos diseños en sus piezas. El trabajo de artesano tiene una conexión directa con el rescate de la identidad cultural, la pertenencia histórica y el estrechamiento del vínculo familiar. En su 
práctica se reconoce que existe un cariño, un amor por lo que se hace, afecto que se ve acentuado por el reconocimiento en la alteridad del trabajo plasmado en cada una de las piezas y diseños de éstas. Su práctica social es aquella que rescata lo natural, con la que se vuelve al origen, aquella que busca la hermosura en lo diverso, no en lo homogéneo y que, por lo tanto, es altamente creativa, detallista, observadora e indagadora. Podemos afirmar además, que la artesanía es un trabajo no profesional, o sea, que siempre es aprehendido de alguna forma, ya sea a través de la experiencia familiar o por tutores, pero que se distingue de las profesiones modernas, en el sentido weberiano de éstas (Aguayo, 2006).

Los artesanos y artesanas han tomado conciencia de revalorar lo original, preservar lo único, lo exclusivo de sus piezas. Los invito a revisar el siguiente pasaje de un artesano de la comuna de Nueva Imperial: "Las artesanías no se pueden estandarizar, porque resulta que es como una enfermera, todas las enfermeras pinchan diferente, entonces el artesano, siempre le va a colocar un matiz diferente a su producto, independientemente de que este sea un mismo diseño”, analogía que nos permite constatar algunas de las características esenciales del trabajo artesanal.

En el camino descubrimos las particularidades de distintos oficios artesanales:

1. Oficio del Retrafe (platería): O el oficio solitario, por el celo que se produce por el uso del saber (técnica). Caracterizado por el trabajo con metales, principalmente la plata, materia prima de difícil y costosa obtención. Su saber está relacionado al fundido y el laminado de los metales y sus diseños son creaciones ligadas a imágenes y representaciones del retrafe con su historia y cultura.

2. Oficio de Textilería: Que se caracteriza por el compromiso en el trabajo comunitario y su saber traspasado al interior de la familia. Su saber está asociado al lavado, escarmenado, hilado, teñido y tejido de la lana, tanto con palillo como con telares (Witran). En uno de los grupos focales se nos dice: "Nosotras lavamos la lana, la hilamos, tejemos y después si tenemos suerte la vendimos, (risas) y si no, no importa” (Artesanas Temuco)

3. Oficio de Tallador en madera: Los escultores del Kimün (conocimiento) Mapuche. Asociado principalmente a la elaboración de los instrumentos musicales ceremoniales Mapuche, tales como: Kultun, Trutruca, Pifilcas y Bombos.

El saber del artesano es semejante al del artista, y los artesanos así lo describen como aquel saber que es único e irrepetible en cada obra que crean, por tanto, que soporta un significado profundo. Los saberes de los oficios artesanales se forjan en un proceso de profunda meditación del artesano con la técnica utilizada. Tanto la técnica del hilado, teñido y tejido, como la del fundido y moldeamiento del metal, como el del trabajo con la madera. 
Los oficios son construidos socialmente y, por lo tanto, no pueden ser vistos como el resultado el producto final de un proceso, puesto que en ellos también se da una significación que implica una forma de valorar la vida que los propios sujetos construyen. Hemos visto que el trabajo para artesanas y artesanos es un elemento constructor de identidad, que les permite definirse como pertenecientes a un lugar determinado, construir subjetividades específicas y situadas. Estas subjetividades son las que queremos revalorizar si queremos pensar en la definición y reflexión de un nuevo modelo de desarrollo. La resignificación del desarrollo a partir de la resignificación de los oficios contemporáneos y tradicionales. Hacer un replanteamiento crítico respecto el proceso contrario al trabajo que ha predominado en el actual modelo de desarrollo, la alienación. Las instituciones financieras y las grandes empresas (nacionales y trasnacionales) se fortalecen al punto de subordinar las economías locales, y sobre todo, debilitando a los Estados, que aparecen cada vez como más impotentes para llevar a adelante proyectos de desarrollo al servicio de sus comunidades.

La artesanía mantiene en variados aspectos contradicciones con lo que entendemos por trabajo en la actualidad. Desde nuestras primeras observaciones del trabajo de campo emergieron elementos que se transformarían en antecedentes dignos de mencionar en esta investigación. El trabajo artesanal es altamente creativo e investigativo, los artesanos regulan su trabajo a través de la observación e indagación de nuevas formas y técnicas. Éste se debate entre un tipo de racionalidad de la interacción social y la racionalidad instrumental. Los artesanos constituyen una "comunidad de oficio", en el sentido que son un grupo social definido por sus interrelaciones específicas, regulares y cotidianas, su cultura compartida y su arraigado sentido de pertenencia que configura una identidad en torno al trabajo ejercido.

La investigación terminó como una forma de análisis del Trabajo, definido como el ejercicio humano de la creación y la producción que en la modernidad se explica por su contrario, la alienación, y una propuesta de análisis de las distintas comunidades de oficios que se conocen. Los oficios, como la forma específica de la profesión aprehendida y, los saberes, como el arte del uso de la técnica. Presentándose como una propuesta teórica para el abordaje de las distintas prácticas sociales del trabajo o de las distintas comunidades de oficio. El telón de fondo fue el hecho de que estas prácticas sociales del trabajo, en este caso la artesanal, nos ayuda a resignificar el desarrollo desde una perspectiva comunitaria.

Redefinir el desarrollo, desde el ámbito del trabajo, significa instalar y rescatar las construcciones sociales alternativas a las que conocemos. Aspectos centrales para alcanzar aquello, según los datos recabados, pueden ser: La posibilidad del alcance de metas personales, los espacios para la innovación en la práctica del trabajo, el reconocimiento en la alteridad y la regulación social de la forma específica que debe adoptar el trabajo al interior de los mercados. En este punto es válido plantear entonces que "un desarrollo generalizado solamente es posible interviniendo en los merca- 
dos, de manera que quien pierda en la competencia no sea condenado a muerte... eso debería llevar a la constitución de sistemas locales y regionales de división del trabajo, e inclusive de monedas locales o regionales... su organización podría describirse como una producción simple de mercancías. Estos sistemas locales y regionales de división del trabajo probablemente configuran hoy la única posibilidad realista de devolverles una base estable de vida” (Hinkelammert, 2001: 20-21)

Algunas líneas de acción para proyectos complementarios de las agencias no gubernamentales y gubernamentales, en materia de artesanía, es la organización de iniciativas de exposición de productos. En estas se mantiene el valor de irreproductibilidad de una pieza y al mismo tiempo existe la posibilidad de que se encarguen diseños a pedido. Otra recomendación general sería ubicar y brindar espacios, talleres, para la confección y venta de las piezas artesanales. Lo anterior, claro está, debe ser estudiado para que el posicionamiento de esos espacios se realice en sus medios, nos referimos, en los campos si así se requiere o en la ciudad donde los propios artesanos consideren necesario.

Repensar el desarrollo, requiere repensar los oficios y el proceso del trabajo. Repensar el oficio del profesor, músico, investigador, interventor, obrero, etc. en términos de una relación contractual social y comunitaria pero también como una práctica de creación artística en movimiento. El desarrollo se ha basado exclusivamente en avanzar en un sistema de conocimiento, es decir, el correspondiente al del occidente moderno. La predominancia de este sistema de conocimiento ha dictaminado la marginación y descalificación de los sistemas de conocimiento no occidentales. En estos últimos podríamos encontrar racionalidades alternativas para orientar la acción social con un criterio diferente a formas de pensamiento economicista y reduccionista (Escobar, 2005).

Entender que, en la práctica, los oficios son una de las formas mediante los cuales los seres humanos nos generamos la posibilidad de atribuirle sentido a nuestras vidas, para poder movernos, de alguna manera entender la materialidad de nuestra existencia, pero también autoconferimos identidad. Que el trabajo es algo esencial para los seres humanos, porque mediante de éste, las personas articulan sus vidas en torno a una labor material. De manera tal, que si los individuos quedan fuera de lo que es el trabajo, se le destruye su mundo no sólo en lo que respecta a lo laboral, sino también a nivel personal y familiar. Para los artesanos un valor determinante es cómo estos se integran y construyen una identidad colectiva por sobre una individual.

\section{Palabras finales}

Al término de este proceso de investigación presentamos un retrato de las prácticas de trabajo de algunos oficios artesanales, en donde observamos algunas características como el rescate de la cultural ancestral, el 
valor implícito en cada una de las piezas, el justo reconocimiento obtenido en la alteridad y la reflexión profunda en el manejo de la técnica.

Podemos concluir que las versiones locales del desarrollo y la modernidad se formulan siguiendo procesos complejos que incluyen las prácticas culturales, de producción, memoria histórica y la ubicación contemporánea dentro de la economía global de bienes y símbolos. La preocupación por los caminos alternativos del desarrollo sigue abierta y se posiciona como un fértil campo de investigación para las ciencias sociales.

Invitamos a la utilización del modelo propuesto para el análisis de las prácticas de trabajo, con el fin describir las distintas comunidades de oficio, en tres niveles de abordaje: el del trabajo, que nos permite una caracterización general de la práctica en términos de su despliegue específico en la sociedad y el contacto con las instituciones; el del oficio, que permite ahondar en la descripción sensible de las distintas formas, tradicionales y modernas, de producir; y el saber, que apunta a explorar y conocer las significaciones profundas del manejo de la técnica y los instrumentos. Una reflexión dialéctica entre estos niveles permitirá sensibilizar conceptos y construir una mirada distinta del proceso de producción. 


\section{Bibliografía}

Aguayo, Cecilia (2006), Las profesiones modernas. Dilemas de conocimiento y poder, Universidad Tecnológica Metropolitana, Santiago de Chile.

Bhabha, Homi (1994), El lugar de la cultura, versión digital disponible en http://flacsocultura.blogspot.com/2007/08/homi-bhabha.html

Boisier, Sergio (2001), Desarrollo (local): ¿De qué estamos hablando?, versión digital disponible en http://www.cedet.edu.ar/sitio/administracion/ agenda/boisier.pdf

Castro Gómez, S. \& Grosfoguel, R (2007), El giro decolonial, Siglo del Hombre editores.

CiPYME. (2007), La PyME en cifras. Informe caracterización regional provincial de la PyME.

Certeau, Michel de. (1999), La invención de lo cotidiano, Universidad Iberoamericana, México.

Di Filippo, A. (2007), "La escuela latinoamericana del desarrollo: Tensiones epistemológicas de un movimiento fundacional”, en Revista Cinta de Moebio 29: 124-154.

Duhart, Daniel (2006), “Exclusión, poder y relaciones sociales”, en Revista $M A D N^{\circ} 14$. Departamento de Antropología, Universidad de Chile.

Elizalde, Antonio (2005), “Desarrollo”, en Salas, R. (coord). Pensamiento crítico latinoamericano. Vol. I (pp. 153-166), Ediciones UCSH, Santiago de Chile.

Escobar, Arturo (1998), La invención del tercer mundo, Fondo de Cultura Económica.

Ídem (2005), “El postdesarrollo como concepto y práctica social”, en Daniel Mato (coord) Políticas de economía, ambiente y sociedad, en tiempos de globalización. pp. 17-31. Facultad de Ciencias Económicas y Sociales, Universidad de Venezuela, Caracas.

Garretón, Manuel Antonio (2000), Política y sociedad entre dos épocas. América Latina en el cambio de siglo, Homo Sapiens ediciones, Rosario.

Glaser, B., \& Strauss, A. (1967), The discovery of grounded theory: strategies for qualitative research, Aldine, New York.

Hopenhayn, Martín (2001), Repensar el trabajo: Historia, profusión y perspectivas de un concepto, Editorial Norma, Buenos Aires. 
Instituto Nacional de Estadísticas (INE) (2009), Informe Económico Regional. Octubre-Diciembre 2008. Versión Digital Disponible en http:// www.ine.cl/canales/chile_estadistico/territorio/iner/2008/pdf/ iercuartotrimestre2008.pdf

Isla, A. \& Colmegna, P. (2007), “La importancia de la cultura y la política en los procesos de desarrollo”, Revista MAD 16: 93-107.

Martínez, Víctor (2006), El enfoque comunitario. El desafío de incorporar a la comunidad en las intervenciones sociales, Ed. Universidad de Chile, Santiago de Chile.

MIDEPLAN (2006), Encuesta de caracterización socioeconómica nacional. Ministerio de planificación.

MIDEPLAN, PNUD (2000), Desarrollo humano en las comunas de Chile. Versión digital disponible en www.pnud.cl.

Programa de las Naciones Unidas para el Desarrollo (PNUD), Universidad de la Frontera (UFRO) y Ministerio de Planificación y Cooperación (MIDEPLAN) (2003), El Índice de desarrollo humano en la población mapuche de la Región de la Araucanía (Una aproximación a la equidad interétnica e intraétnica), Temas de Desarrollo Humano Sustentable nº. PNUD, Santiago.

Strauss, A.L. \& Corbin, J (1990), Basics of qualitative research, Sage.

Valles, Miguel (1999), Técnicas cualitativas de investigación social. Reflexión metodológica y práctica profesional, Síntesis. 